\title{
Solid state atomic processors for light
}

\section{Crozatier \\ G. Gorju}

V. Lavielle

\section{A. Louchet}

F. Bretenaker

T. Chanelière

F. Goldfarb

\section{Lorgeré}

\section{J.-L. Le Gouët}

jean-louis.legouet@lac.u-psud.fr

\begin{abstract}
Laboratoire Aimé Cotton, CNRS UPR3321, Univ Paris Sud
bâtiment 505, campus universitaire, 91405 Orsay cedex

Laboratoire Aimé Cotton, CNRS UPR3321, Univ Paris Sud

bâtiment 505, campus universitaire, 91405 Orsay cedex

Laboratoire Aimé Cotton, CNRS UPR3321, Univ Paris Sud

bâtiment 505, campus universitaire, 91405 Orsay cedex

Laboratoire Aimé Cotton, CNRS UPR3321, Univ Paris Sud

bâtiment 505, campus universitaire, 91405 Orsay cedex

Laboratoire Aimé Cotton, CNRS UPR3321, Univ Paris Sud

bâtiment 505, campus universitaire, 91405 Orsay cedex

Laboratoire Aimé Cotton, CNRS UPR3321, Univ Paris Sud

bâtiment 505, campus universitaire, 91405 Orsay cedex

Laboratoire Aimé Cotton, CNRS UPR3321, Univ Paris Sud

bâtiment 505, campus universitaire, 91405 Orsay cedex

Laboratoire Aimé Cotton, CNRS UPR3321, Univ Paris Sud

bâtiment 505, campus universitaire, 91405 Orsay cedex

Laboratoire Aimé Cotton, CNRS UPR3321, Univ Paris Sud

bâtiment 505, campus universitaire, 91405 Orsay cedex
\end{abstract}

This paper is devoted to optics in rare earth ion doped crystal at low temperature. In cryogenic conditions, interesting features come from absorption rather than from transparency. The optical transition linewidth is considerably reduced, which also corresponds to a strong increase of quantum state lifetime. Linewidth narrowing leads to signal processing applications. Specific use for RADAR warning receivers is considered here. Then the quantum lifetime extension is illustrated by coherent transient processes that represent necessary experimental steps on the way to quantum information research. [DOI: 10.2971/jeos.2008.08025]

Keywords: RF signal processing, rare earth ion doped crystal, spectral hole burning, coherent transients, quantum information

\section{INTRODUCTION}

Solid state materials are extensively used as non-linear optical media. In those applications the non-linear susceptibility is the key parameter. Other applications take advantage of the long excited state lifetime in embedded impurities such as rare earth ions. Those impurities can play the role of energy tanks for light amplification. Erbium-doped fibers offer a good illustration of such amplifiers in optical telecommunications. Rare earth ion doped fibers or crystals are used as gain media in various lasers. All these room temperature properties enjoy a widespread usage. On the contrary, cryogenic temperature properties, such as narrow linewidth, are still largely ignored, although they are common in well known optical materials such as rare earth ion-doped crystals (REIC). Recently, the development of energy-efficient and compact mechanical cryocoolers [1] has renewed the interest in low temperature applications. Since narrow linewidth means long quantum state lifetime, basic science related to quantum information also benefits from the attention paid to low temperature conditions.
When absorbing centers are embedded in a host matrix and the medium is cooled to a few degrees Kelvin, homogeneous absorption line widths are dramatically reduced as a result of strongly diminished environmental fluctuations. While the cooling decreases the homogeneous widths, it has little effect on the inhomogeneous broadening that results from the static interaction of the absorbing centers with the host. When exposed to monochromatic light, the material is excited only within the homogeneous-width of the incoming light and a narrow spectral hole is burned at this position within the absorption profile [2]. In the long quest for practical applications of spectral hole burning (SHB) in solid materials, optical storage was considered first. The goal was to overcome the storage density diffraction limit by adding a fourth non-spatial dimension, namely frequency, to the memory volume. Despite excellent performance in terms of storage density and capacity, access time and transfer rate, SHB memories suffer from operating at liquid helium temperature, a severe limitation for mass market application. Cryogenics has to be balanced 
by an unquestionable technological bonus. It was soon realized that the specific time/frequency dimension should not be considered on an equal footing with the spatial ones, as it was in large memory application. Instead, the inhomogeneous linewidth can be regarded as the spectral bandwidth of an optically carried signal analog processor. In this respect SHB materials can outdo any existing electronic processor and appear to be potentially attractive microwave photonics components, offering an instantaneous bandwidth in excess of $10 \mathrm{GHz}$ with a channel capacity larger than $10^{4}$. This can be used for range and Doppler processing of RADAR return signals. The crosscorrelation function of the encoded RADAR pulse and the return signal is stored as a spectral grating within the SHB material and retrieved by absorption spectroscopy [3]. Another application has been considered for array antennas. The achromatic true time delays of the array components are stored in a SHB material and are applied to arbitrary waveform optically carried signals without latency, either at transmission or reception [4]-[7]. In this paper we review our demonstrations of SHB capabilities for broadband spectral analysis with unity probability-of-intercept. Two different approaches have been considered. The first one uses the SHB material as a storage medium where the optically carried RF signal spectrum is accumulated before readout. Unlike this spectral photography scheme, the second approach avoids the storage of the signal under investigation in the SHB medium. Instead the material is programmed as an analog processor that continuously processes the signal-carrying beam. Both architectures are implemented in a $\mathrm{Tm}^{3+}$ :YAG crystal.

The same $\mathrm{Tm}^{3+}$-doped material offers promising prospects for quantum information storage. Transport of quantum information is ideally accomplished by light but, at some stage, a material system is needed for processing and/or storage. Resonant excitation of an atomic transition provides appropriate strong coupling between light and matter. However, interaction with a single atom is not enough to trap the incident photon with absolute certainty. One can increase the coupling by placing the atom inside a high finesse cavity. Alternatively, light can be trapped in a macrocopic ensemble of atoms. So far, the mapping of a quantum state of light onto an atomic ensemble has been implemented in atomic vapors [8] and cold atom clouds [9]. The extension to solid state media has been actively pursued. In this context, REIC have been proposed as candidates for optical quantum storage [10]-[13]. To some extent, REIC at low impurity concentration are similar to atomic vapors with the advantage of no atomic diffusion. Storing information is not enough. One also need interrogate the memory at will, controlling the moment when the signal is restored. This can be achieved through an auxilliary optical transition, coupled to the quantum field capture transition. The $\Lambda$ three-level system is consistent with such a protocol. In this system a common upper level links the two transitions that are connected to two sub-levels of the electronic ground state. Therefore, in a simple way, the transition to be excited by the quantum field can be triggered for storage or restitution by an external control field. The quantum state of light is stored in a superposition state of the ground state sublevels. In this paper we report on our recent investigation of this superposition lifetime in $\mathrm{Tm}^{3+}$ :YAG.

\section{RF SIGNAL SPECTRAL ANALYSIS}

\subsection{Spectrum photography architecture}

Although it has been considered only recently [14]-[16], the spectrum photography architecture is actually the simplest of the two architectures we consider here.

The RF signal to be analyzed is first transposed on a monochromatic fixed frequency laser beam with the help of a Mach-Zehnder modulator (see Figure 1). The laser frequency is adjusted in such a way that one of the RF-signal-carrying side-bands lies inside the SHB material absorption band. Upon crossing the SHB material, the beam excites the ions that are resonant with the different optically carried spectral components of the RF signal. Consequently, the optically carried RF spectrum is recorded in the material absorption spectrum. By probing the material transmission with a frequency chirped laser one readily obtains a temporal image of the engraved spectrum. Although the read out scanning procedure is reminiscent of usual electronic spectrum analyzers, the SHB analyzer exhibits a very distinctive feature. Indeed, although spectral addresses are probed sequentially the RF signal is continuously recorded in the SHB material that operates as a spectral buffer memory. By simply using two separate lasers for engraving and probing one may reach unity probability-of-intercept. Storage lifetime in the buffer memory is limited by the excited level population lifetime that can exceed $10 \mathrm{~ms}$ in the materials we consider. The spectral resolution is limited by the laser noise and the square root of the chirp rate of the reading laser. Given the broad bandwidth to be scanned in less than $10 \mathrm{~ms}$, the chirp rate limited resolution will dominate the homogeneous linewidth of the optical transition. The geometry of the experiment can be rather simple, allowing us to consider this experiment as an elementary pixel of a future broadband spectrally resolved $\mathrm{RF}$ imaging system. We have implemented this protocol in a $\mathrm{Tm}^{3+}$ :YAG crystal. An example of experimental data is displayed in Figure 2.

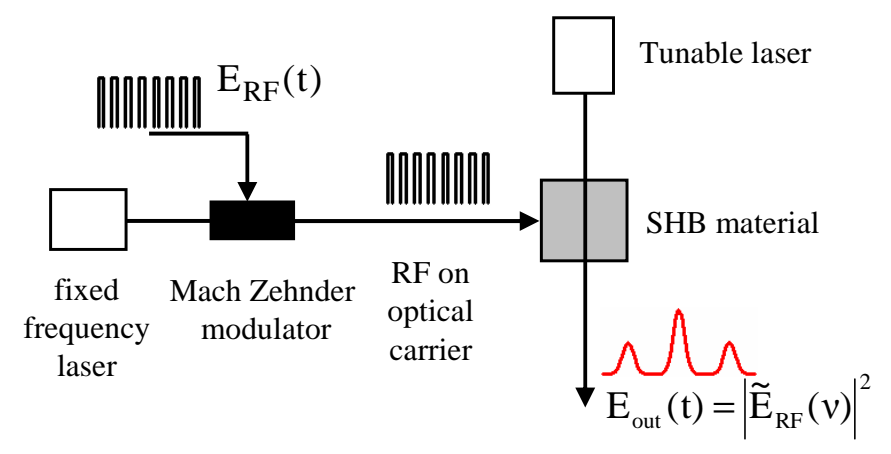

FIG. 1 Block diagram of the spectrum photography analyzer. The fixed frequency laser carries the RF spectrum to engrave it in the crystal absorption spectrum. The tunable laser is used simultaneously to probe the crystal absorption, thus revealing the stored RF spectrum.

\subsection{Rainbow analyzer}

The rainbow analyzer concept relies on the engraving of monochromatic gratings in a frequency selective material. Each grating is able to diffract a single spectral component, 


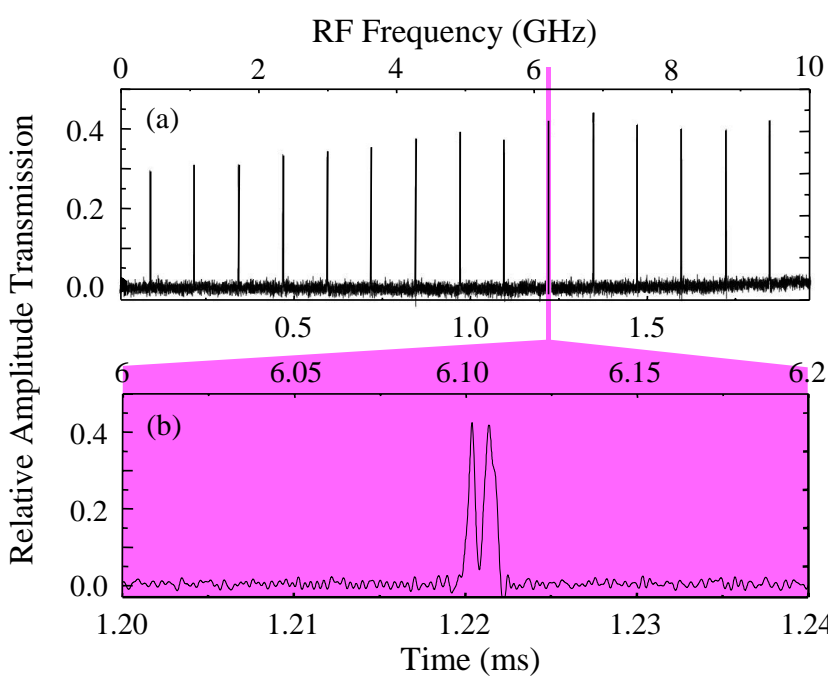

FIG. 2 (a) Example of experimentally observed RF spectrum. The $10 \mathrm{GHz}$ bandwidth readout is performed in $2 \mathrm{~ms}$. Fifteen equally spaced tones haved been engraved in the crystal, except for a $5 \mathrm{MHz}$ doublet, which is perfectly resolved as can be seen in (b).

with a resolution ultimately determined by the homogeneous linewidth of the medium, which is usually less than $1 \mathrm{MHz}$ at the temperature of $5 \mathrm{~K}$. A large number of gratings can coexist within the inhomogeneous width of the absorption line, which may reach tens of GHz. By varying the laser frequency synchronously with the angle of incidence during the engraving procedure, one associates a specific diffraction angle with each specific spectral component. Therefore, the different spectral components of an incident polychromatic probe beam are diffracted and simultaneously retrieved in different directions. The stack of monochromatic gratings works as a spectrometer which is expected to exhibit a resolution of less than $1 \mathrm{MHz}$ and a bandwidth of several tens of GHz. As depicted in Figure 3, radiofrequency spectral analysis can be performed after transfer of the test microwave signal to an optical carrier with the help of a Mach-Zehnder electro-optic modulator $(\mathrm{MZM})$. The bandwidth of the frequency selective medium indeed matches that of integrated MZMs developed for high flow telecommunication. To be more specific, frequency selective media can be devised to cover a bandwidth in excess of $50 \mathrm{GHz}$ offered by fast integrated MZMs [17]. Since the

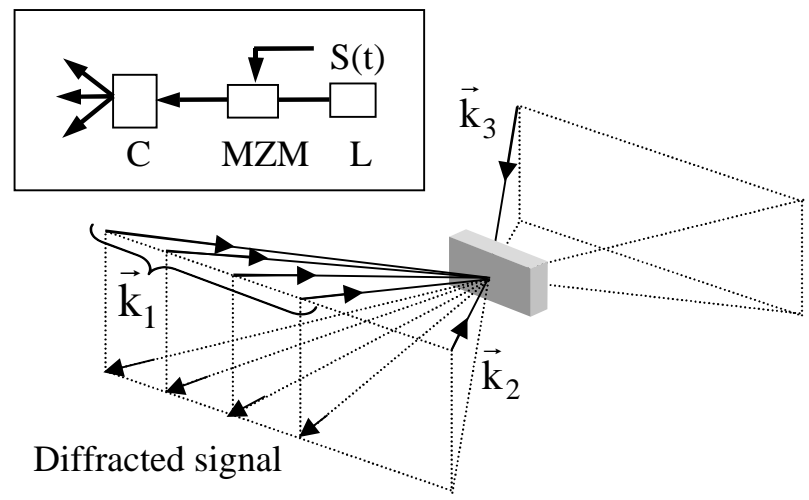

FIG. 3 Rainbow spectrometer concept. Block-diagram in inset. Mach-Zehnder modulator(MZM), RF signal $(S(t))$, laser (L), crystal (C). The $3 \mathrm{D}$ picture shows the counter propagating box geometry giving independent access to the engraving(\#1, \#2), readout (\#3) and diffracted beams. spectrometer relies on the angular separation of the frequency components, the number of frequency channels equals the number of different angular directions that can be addressed by the device. For a given setup this number is fixed. However, the engraving laser frequency scanning range, which determines the spectrometer bandwidth, can be varied easily. By reducing this range while the channel number is kept fixed, one is able to zoom in on a specific spectral region with improved spectral resolution. Since energy is detected in each spectral channel, this processor is independent of the spectral coherence features of the channeled signal, quite in the same way as the spectrum photography architecture. The potential of a similar arrangement to process the spectral phase and operate as a time-integrating correlator has been addressed in [18]

The rainbow analyzer is reminiscent of the well known acousto-optic spectrometer. In the latter device a Bragg cell achieves two functions. It both transfers the RF signal on the optical carrier and accomplishes the angular separation of the optically carried spectral components. Acoustic wave absorption limits the bandwidth to $\sim 1 \mathrm{GHz}$. The integrated MZM offer a much larger bandwidth. However a MZM transfers only the RF signal on the optical carrier but does not achieve the angular separation of the spectral components, since the carrier and the side bands propagate along the same direction. In the SHB spectrometer, the SHB crystal is intended to complement the MZM component by providing the angular separation.

The beam configuration is strongly constrained by the material limitations. Owing to the short lifetime of the engraving, the gratings must be refreshed continuously and simultaneously diffract the impinging signal beam. This condition is satisfied in the box configuration presented in Figure 3. The probe beam that carries the RF signal to be processed propagates along $\vec{k}_{3}$. It lies out of the plane defined by the engraving beam wave vectors $\vec{k}_{1}$ and $\vec{k}_{2}$. This non-coplanar arrangement is consistent with simultaneous writing and readout since the diffracted beam is directed along $\vec{k}_{3}+\vec{k}_{1}-\vec{k}_{2}$ which differs from all the incident wave vectors. The wave vectors $\vec{k}_{2}$ and $\vec{k}_{3}$ are headed in fixed directions while $\vec{k}_{1}$ rotates synchronously with the frequency scan of the engraving beams. Therefore the different spectral components that are carried by the probe beam are diffracted in different directions. The box configuration satisfies the phase matching condition, $\vec{k}_{1}-\vec{k}_{2}$ being orthogonal to $\vec{k}_{3}+\vec{k}_{2}$. With the rainbow architecture, our best achievement so far has been $3.3 \mathrm{GHz}$ instantaneous bandwidth with 100-channel resolution (see Figure 4). The programmed material was again a $\mathrm{Tm}^{3+}: \mathrm{YAG}$ crystal [19]. In this experiment we simulated a multiple-line RF signal by making the laser perform various discrete frequency jumps during the readout step of the writing/readout sequence. In a previous publication [20] we demonstrated the spectral analysis of a true RF signal that was put on the probe beam with the help of a Mach-Zehnder modulator.

To meet the requirements of both architectures in terms of stability, scanning speed, range and repeatability, original tunable lasers have been developed [21,22] together with specific diagnostic, monitoring and control techniques [23]-[25]. 


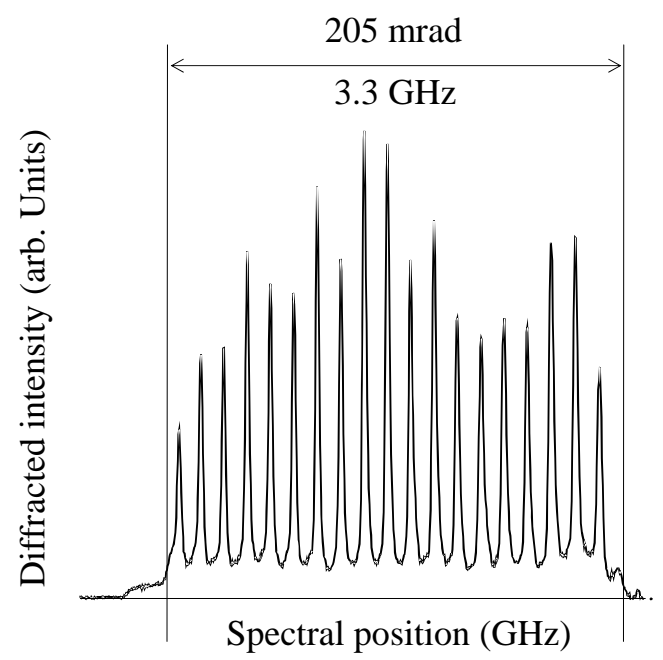

FIG. 4 Rainbow spectrometer demonstration. The simulated multi-frequency RF signal undergoes 19 successive $175 \mathrm{MHz}$-wide jumps, and dwells only 10ţs at each spectral position. The observed resolution is consistent with 100-channel capacity.

\subsection{Distinctive features of each architecture}

Both architectures offer at least $10 \mathrm{GHz}$ detection bandwidth and unity probability-of-intercept. In both devices a monochromatic laser has to be scanned repeatedly over the analyzed bandwidth. Nonetheless each architecture exhibits specificic properties. The spectral photography approach combines an unsurpassed channel capacity with a rather simple optical design. In this design the REIC works as a buffer memory, which saves all the available information but does not give immediate and simultaneous access to all the spectral components. The access time to the stored data is limited by both the readout laser scanning rate and the detector bandwidth. In the reported experiments this rate does not exceed $10 \mathrm{GHz} / \mathrm{ms}$. Besides the buffer memory exhibits a fixed lifetime of $10 \mathrm{~ms}$. This can also set an upper limit to the repetition rate. In the rainbow analyzer the access time is not limited by the optical protocol. Indeed the incoming signal is instantaneously split into its spectral components by the programmed crystal. The scanned laser is not used to readout but to refresh the crystal programming. The scan rate is not related to the access time but to the stored programming lifetime. The demonstrated $10 \mathrm{GHz} / \mathrm{ms}$ chirp rate is consistent with the $10 \mathrm{~ms}$ memory lifetime. On the downside, the rainbow analyzer cannot offer more than a few hundred spectral channels and suffers from a rather complex optical design.

\section{OPTICAL EXCITATION OF NUCLEAR SPIN COHERENCE}

Most Quantum Memory for Light (QML) protocols rely on the transfer of a quantum state of light into a long-lived spin coherence that is free from decoherence via spontaneous emission. This can be achieved in a $\Lambda$-type three-level system where two hyperfine or spin sublevels are optically connected to a common upper level. It is noteworthy that the essence of $\Lambda$-system operation, namely the optical excitation of nuclear spin, has been practised in REIC for almost 30 years, but always in praseodymium- or europium-doped crystals. We recently demonstrated the existence of a $\Lambda$-system with adjustable ground state splitting in thulium-doped YAG [26, 27]. Several features make the trivalent thulium ion attractive. First, its $793 \mathrm{~nm}$ wavelength falls within reach of easily stabilized semiconductor lasers, unlike Pr and Eu. Then, its $I=$ $1 / 2$ nuclear spin gives rise, under external magnetic field, to a simple straightforward 4-level optical system in which a $\Lambda$ system can be selected. Moreover, because of this simple structure, the sublevel splitting can be controlled easily by the external magnetic field. This crystal could therefore be used as an atomic quantum memory adapted to the bandwidth of existing quantum sources, unlike Pr- or Eu-doped compounds where the hyperfine structure is fixed and small. For these reasons, thulium-doped materials are favorable alternatives to Pr- and Eu-doped compounds for quantum storage applications, with the triple advantage of a tractable wavelength, a simple level system and an adjustable ground state splitting.

We turn now to investigating the ground state spin coherence with optical means. Initially the two ground state sublevels are equally populated and the optical transition frequency is distributed over a $25 \mathrm{GHz}$-wide inhomogeneously broadened absorption profile. Within this huge bandwidth, we select a narrow interval over which we prepare the ions by pumping them into a single sublevel. This way, the two-photon excitation is optimized. Then we excite the ground state spin coherence with a bichromatic laser pulse that is comprised of two components at frequencies $\omega_{1}$ and $\omega_{2}$. The two-photon excitation at frequency $\omega_{1}-\omega_{2}$ is tuned to resonance with the spin transition at $\Delta_{g}$, the ground state splitting frequency. If all atoms remain phased together, one could monitor the spin coherence evolution by coherent forward Raman scattering: a long rectangular monochromatic pulse at frequency $\omega_{1}$ excites one transition of the $\Lambda$ and converts part of the spin coherence into an optical coherence along the other transition. The resulting optical emission could be detected as a beatnote at frequency $\Delta_{g}$ against the probe pulse. Unfortunately, the spin transition is inhomogeneously broadened, which leads to spin coherence dephasing. This makes the beatnote vanish on the timescale of the excitation pulse duration. In order to recover the optical signature of the spin coherences, we resort to the Raman echo procedure [28]-[32]. We apply an additional bichromatic pulse, resonant with the two-photon transition, at mid-time between initial excitation and final probing. This pulse reverses the spin coherence time evolution, so that, at the moment of probing, the spin coherences are phased together again and give rise to an optical emission (see Figure 5). The Raman echo signal is observed by means of real-time Fast Fourier Transform (see Figure 6) [33].

\section{CONCLUSION}

As illustrated by these few examples, resonant interaction of light with atomic transitions in a REIC offers a variety of perspectives, ranging from electronic warfare applications to quantum information basic research. The same material can be regarded as an optical component to be hybridized with fast electronic, or as a sample similar to a laser cooled atom cloud. The RF spectral analysis architectures have demon- 


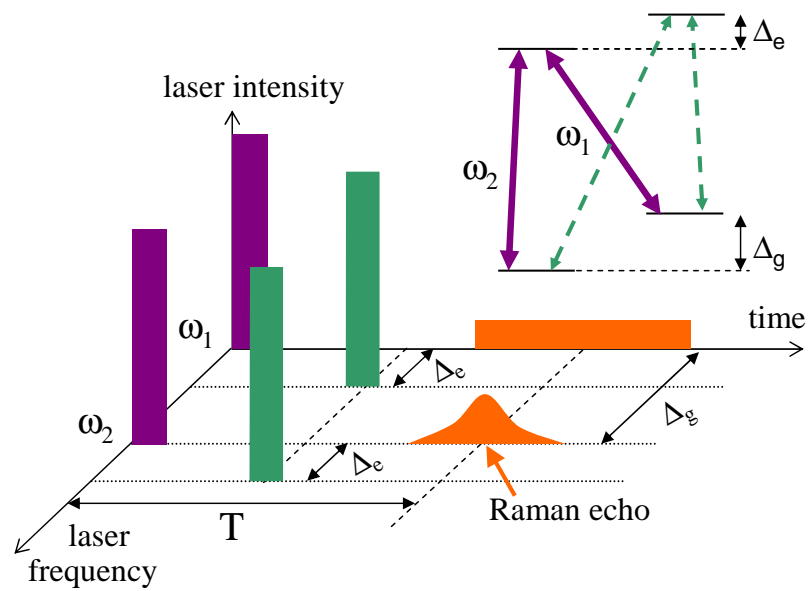

FIG. 5 (color online) Raman echo pulse sequence. An auxilliary $\Lambda$-system, connected to the other upper-state sub-level, is used for photon echo elimination. The second pulse is detuned from the first one by the excited state splitting $\Delta_{e}$, still satisfying the two-photon resonance. Inset: 4 -level system. The first and second pulse frequencies $\left(\omega_{1}, \omega_{2}\right)$ and $\left(\omega_{1}+\Delta_{e}, \omega_{2}+\Delta_{e}\right)$ are respectively depicted in solid and dashed arrows.

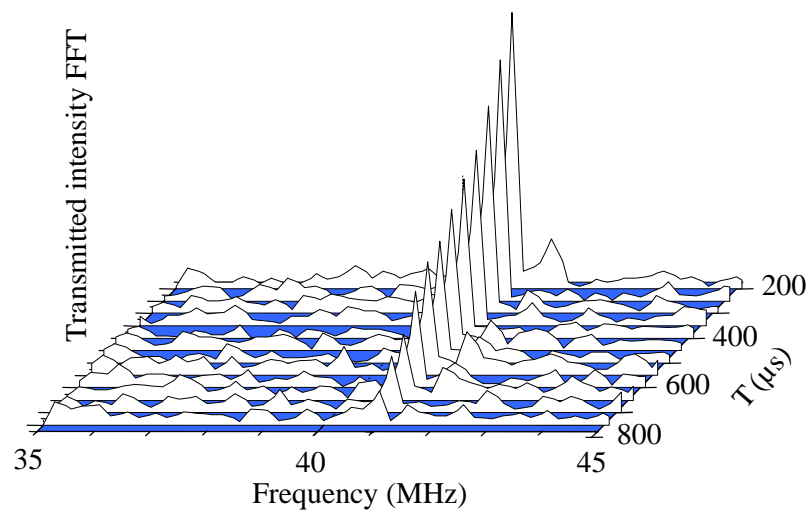

FIG. 6 (color online) Raman echo spectrum for $\Delta_{g}=41 \mathrm{MHz}$ as a function of the delay $T$. The spin coherence lifetime is derived from the exponential decay of the signal.

strated unrivaled capabilities in terms of instantaneous bandwidth, number of frequency channels, and probability-ofintercept. On the quantum information side, the optical driving and detection of nuclear spin coherence represents a modest step toward a quantum memory for light but the REIC material behaves as a fully functional three-level atom assembly.

\section{References}

[1] R. G. Ross, Jr., R. F. Boyle, R. W. Key, and D. R. Coulter, Proc. SPIE 4850, 1020-1028 (2003).

[2] A. Szabo, Phys. Rev. Lett. 27, 323-326 (1971).

[3] Z. Cole, T. Böttger, R. Krishna Mohan, R. Reibel, W. R. Babbitt, R. L. Cone, and K. D. Merkel, Appl. Phys. Lett. 81, 3525-3527 (2002).

[4] K. D. Merkel, and W. R. Babbitt, Opt. Lett. 21, 1102-1104 (1996).

[5] K. D. Merkel, Z. Cole, and W. R. Babbitt, J. Lumin. 86, 375-382 (2000).

[6] L.-M. Duan, M. Lukin, J. I. Cirac, and P. Zoller, Nature 414, 413-418 (2001)

[7] R. Reibel, Z. Barber, M. Tian, and W. R. Babbitt, Opt. Lett. 27,
494-496 (2002).

[8] B. Julsgaard, A. Kozhekin, and E. S. Polzik, Nature (London) 413, 400 (2001).

[9] A. Kuzmich, W. P. Bowen, A. D. Boozer, A. Boca, C. W. Chou, L.-M. Duan, and H. J. Kimble, Nature (London) 423, 731 (2003).

[10] A. V. Turukhin, V. S. Sudarshanam, M. S. Shahriar, J. A. Musser, B. S. Ham, and P. R. Hemmer, Phys. Rev. Lett. 88, 023602 (2002).

[11] J. J. Longdell, E. Fraval, M. J. Sellars, and N. B. Manson, Phys. Rev. Lett. 95, 63601 (2005).

[12] A. L. Alexander, J. J. Longdell, M. J. Sellars, and N. B. Manson, Phys. Rev. Lett. 96, 043602 (2006).

[13] S. Hastings-Simon, M. Staudt, M. Afzelius, P. Baldi, D. Jaccard, W. Tittel, and N. Cisin, Opt. Commun. 266, 716 (2006).

[14] M. Colice, F. Schlottau, K. Wagner, K. Mohan, W. R. Babbitt, I. Lorgeré, and J.-L. Le Gouët, Proc. SPIE 5557, 132-139 (2004).

[15] G. Gorju, V. Crozatier, I. Lorgeré, J.-L. Le Gouët, and F. Bretenaker, IEEE Photonic Tech. L. 17, 2385-2387 (2005)

[16] F. Schlottau, M. Colice, K. H. Wagner, and W. R. Babbitt, Opt. Lett. 30, 3003-3005 (2005).

[17] Y. Sun, C. W. Thiel, R. L. Cone, R. W. Equall, and R. L. Hutcheson, J. Lumin. 98, 281-287 (2002).

[18] F. Schlottau, and K. Wagner, J. Lumin. 107, 90-102 (2004).

[19] V. Lavielle, F. De Seze, I. Lorgeré, and J.-L. Le Couët, J. Lumin. 107, 75-89 (2004).

[20] V. Lavielle, I. Lorgeré, J.-L. Le Gouët, S. Tonda, and D. Dolfi, Opt. Lett. 28, 384-386 (2003).

[21] L. Ménager, L. Cabaret, I. Lorgeré, J.-L. Le Gouët, Opt. Lett. 25, 1246-1348 (2000)

[22] V. Crozatier, B. K. Das, G. Baïli, G. Gorju, F. Bretenaker, J.-L. Le Gouët, I. Lorgeré, and W. Sohler, IEEE Photonic. Tech. L. 18, 15271529 (2006)

[23] G. Gorju, V. Crozatier, I. Lorgeré, J.-L. Le Gouët, and F. Bretenaker, Eur. Phys. J. Appl. Phys 30, 175-183 (2005).

[24] V. Crozatier, G. Gorju, F. Bretenaker, J.-L. Le Gouët, I. Lorgeré, C. Gagnol, and E. Ducloux, Appl. Phys. Lett. 89, 261115 (2006).

[25] G. Gorju, A. Jucha, A. Jain, V. Crozatier, I. Lorgeré, J.-L. Le Gouët, and F. Bretenaker, Opt. Lett. 32, 484 (2007).

[26] F. de Seze, A. Louchet, V. Crozatier, I. Lorgeré, F. Bretenaker, J.L. Le Gouët, 0. Guillot-Noël, and Ph. Goldner, Phys. Rev. B 73, 085112 (2006).

[27] A. Louchet, J. S. Habib, V. Crozatier, I. Lorgeré, F. Goldfarb, F. Bretenaker, J.-L. Le Gouët, 0. Guillot-Noël, and Ph. Goldner, Phys. Rev. B 75, 035131 (2007).

[28] S. R. Hartmann, IEEE J. Quantum Elect. 4, 802 (1968).

[29] P. Hu, S. Geschwind, T. M. Jedju, Phys. Rev. Lett. 37, 1357 (1976).

[30] K. P. Leung, T. W. Mossberg, and S. R. Hartmann, Phys. Rev. A 25, 3097 (1982).

[31] B. S. Ham, M. S. Shahriar, M. K. Kim, and P. R. Hemmer, Opt. Lett. 22, 1849 (1997).

[32] A. L. Alexander, J. J. Longdell, and M. J. Sellars, J. Opt. Soc. Am. B 24, 2479 (2007).

[33] A. Louchet, Y. Le Du, F. Bretenaker, T. Chanelière, F. Coldfarb, I. Lorgeré, and J.-L. Le Gouët, 0. Guillot-Noël, and Ph. Goldner, Phys. Rev. B 77, 195110 (2008). 